\title{
A NOTE ON RANK-ONE OPERATORS IN REFLEXIVE ALGEBRAS
}

\author{
CECELIA LAURIE AND W. E. LONGSTAFF
}

\begin{abstract}
It is shown that if the invariant subspace lattice of a reflexive algebra $(t$ '. acting on a separable Hilbert space, is both commutative and completely distributive, then the algebra generated by the rank-one operators of $t^{\prime}$ is dense in $c^{\prime}$ is any of the strong, weak, ultrastrong or ultraweak topologies. Some related density results are also obtained.
\end{abstract}

The main purpose of this note is to clarify the role of the rank-one operators in a reflexive algebra with a (commutative) completely distributive invariant subspace lattice. A complete lattice is completely distributive if it permits distribution of the lattice operations over families of arbitrary cardinality (see below for a precise definition). Every nest, i.e. every totally ordered subspace lattice, is completely distributive. For a subspace lattice, the property of being completely distributive is intimately related to the presence of rank-one operators in the associated reflexive algebra. For a given reflexive algebra $A^{\prime}$, we will refer to the subalgebra generated by the rank-one operators in $\mathcal{G}^{\prime}$ as the rank-one subalgebra of $\mathcal{Q}$. In [10] it was shown that if the rank-one subalgebra of $\mathcal{U}^{\prime}$ is strongly dense in $\mathcal{Q}$ then the lattice of invariant subspaces of $\leftrightarrow$ is completely distributive. In the converse direction, it was known (see [9]) that the rank-one subalgebra of a reflexive algebra with completely distributive invariant subspace lattice was big enough to determine the lattice and the natural question to ask was: is it big enough to determine the algebra? Specifically, is the rank-one subalgebra of a reflexive algebra with completely distributive invariant subspace lattice strongly dense in the algebra? This was known to be true for nest algebras [2] and also in the case where the underlying Hilbert space was finite-dimensional. Subsequently, Lambrou [7] showed that complete distributivity of a subspace lattice implied a condition somewhat weaker than the desired strong density. Here we show that the answer is affirmative if the additional requirement of commutativity is imposed on the invariant subspace lattice. Some related density results are obtained. Specifically, our main result is that if the invariant subspace lattice of a reflexive algebra $\mathcal{Q}$, acting on a separable Hilbert space, is commutative and completely distributive, then the rank-one subalgebra of $\mathbb{A}$ is dense in $\mathbb{Q}$ in any of the strong, weak, ultrastrong or ultraweak topologies. As a consequence, we show that if the invariant subspace lattice of a reflexive algebra $\mathbb{Q}$ is

Received by the editors January 19, 1983. This paper was presented (by the first author) in the Special Session of Operator Algebras in Operator Theory, Annual Meeting of the A.M.S., January 1983.

1980 Mathematics Subject Classification. Primary 47D25. 
commutative, the density of any of a certain family of subsets of $Q$ implies the density of the rank-one subalgebra in $Q$.

Throughout this note all Hilbert spaces will be separable and complex. If $\varrho$ is a collection of (orthogonal) projections acting on a Hilbert space $\mathcal{H}$, we let $\mathrm{Alg} \varrho$ denote the algebra of all (bounded linear) operators on $\mathcal{H}$ which leave invariant each projection in $\mathcal{L}$. If $\mathscr{Q}$ is a collection of operators on $\mathcal{H}$, we let Lat $\mathbb{Q}$ denote the lattice of all projections on $\mathcal{H}$ which are left invariant by every operator in $Q$. The algebra $\mathbb{Q}$ is said to be reflexive if $\operatorname{Alg} \operatorname{Lat} Q=Q$. A subspace lattice on $\mathcal{H}$ is a lattice of projections acting on $\mathscr{H}$ which contains both 0 and $I$ and is closed in the strong operator topology. Every subspace lattice is complete in the sense that it is closed under the formation of arbitrary intersections and arbitrary (closed linear) spans. A subspace lattice is called commutative if it consists of mutually commuting projections. A semi-invariant projection $P$ of $Q$ is a projection of the form $P=E-F$ where $E, F \in$ Lat $Q$ and $F \leqslant E$. For any projection $Q$ we let $Q^{\perp}=I-Q$.

An abstract complete lattice is said to be completely distributive if the following identity and its dual hold for arbitrary index sets:

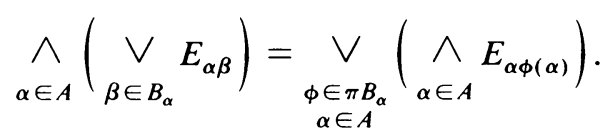

There are many characterizations of complete distributivity. For an extensive discussion, see [6]. For the characterization we will use, we need the following definitions. For a subspace lattice $\mathcal{L}$ and for $M, L \in \mathcal{L}$ define

$$
M_{-}=\vee\{N \mid M \vDash N, N \in \mathcal{L}\}, \quad L_{*}=\wedge\left\{M_{-} \mid M \neq L, M \in \mathcal{L}\right\} .
$$

(We use the conventions $\vee_{\phi}=0$ and $\wedge_{\phi}=I$.) It is easy to see that $L \leqslant L_{*}$, for $L \in \mathcal{L}$. We will use the fact [9] that $\mathcal{L}$ is completely distributive if and only if $L=L_{*}$, for all $L \in \mathcal{L}$.

Proposition 1. Let $\mathcal{L}$ be a completely distributive subspace lattice on a Hilbert space $\mathcal{H}$. Let $P$ be a projection on $\mathcal{H}$ with the property that for every $K \in \mathcal{L}$, either $K \leqslant P^{\perp}$ or $P \leqslant K_{-}$. Then $P=0$.

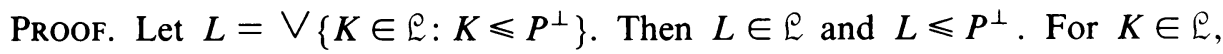
$K \leqslant P^{\perp}$ if and only if $K \leqslant L$. Hence, for $K \in \mathcal{L}, K \leqslant L$ implies that $P \leqslant K$ (using the given property). Since $\mathcal{L}$ is completely distributive,

$$
L=L_{*}=\wedge\left\{K_{-} \mid K \$ L, K \in \varrho\right\},
$$

so $P \leqslant L$. Thus $P \leqslant L \leqslant P^{\perp}$ so $P=0$.

LEMMA 2. Let $\mathcal{L}$ be a subspace lattice on $\mathcal{H}$ and let $\mathcal{Q}=\mathrm{Alg} \mathcal{L}$. Let $\mathcal{R}$ denote the rank-one subalgebra of $Q$. Then $\mathcal{L}$ is completely distributive if and only if $P \Re P \neq 0$ for every nonzero semi-invariant projection $P$ of $\mathbb{Q}$.

Proof. First, suppose $\mathcal{L}$ is completely distributive. We show that $P \Re P \neq 0$ for every nonzero (not necessarily semi-invariant) projection $P$. Let $P$ be a projection 
and suppose $P$ G $P=0$. We show that $P$ has the property described in Proposition 1. Let $K \in \mathcal{L}$ and suppose that $K \leqslant P^{\perp}$. If $K_{-}=I$, then certainly $P \leqslant K_{-}$. Suppose $K_{-} \neq I$. Let $f \in K \mathfrak{H}$ satisfy $f \notin P^{\perp} \mathfrak{H}$ and let $e \in K_{-}^{\perp} \mathfrak{H}$ be nonzero. Then the operator $e \otimes f$, defined by $(e \otimes f) x=(x, e) f(x \in \mathcal{H})$, belongs to $\Re$ [9, Lemma 3.1] and since $P f \neq 0$, we must have $P e=0$. Thus $K_{-}^{\perp} \leqslant P^{\perp}$ so $P \leqslant K_{-}$. By Proposition $1, P=0$.

Conversely, assume that $P \mathscr{R} P \neq 0$ for every nonzero semi-invariant projection $P$ of $\mathcal{Q}$. Let $L \in \mathcal{L}$ and suppose $L \neq L_{*}$. Then $L<L_{*}$ and $P=L_{*}-L$ is a nonzero semi-invariant projection of $Q$. By assumption, there exists a rank-one operator $R$ in (4) such that $\left(L_{*}-L\right) R\left(L_{*}-L\right) \neq 0$. This contradicts the fact that every rank-one operator in $Q$ maps $L_{*}$ into $L$ (see the proof of Theorem 3.1 in [10]). Thus $L=L_{*}$ for every $L \in \mathcal{L}$, so $\mathcal{L}^{*}$ is completely distributive.

THEOREM 3. Let $\mathfrak{L}$ be a commutative completely distributive subspace lattice on a separable Hilbert space $\mathcal{H}$. Then the rank-one subalgebra of $\mathrm{Alg} \mathcal{E}$ is dense in $\mathrm{Alg} \mathcal{E}$ in any of the weak, strong, ultraweak, or ultrastrong topologies.

Proof. Ultraweak density follows from Lemma 2 and the following result $[8$, Theorem 4.1]:

Let $\mathcal{Q}$ be a reflexive algebra on a separable Hilbert space with Lat $\mathcal{Q}$ commutative. Let $\mathcal{Q}_{0}$ be an ideal in $Q$, such that $P Q_{0} P \neq 0$ for all nonzero semi-invariant projections $P$ of $Q^{P}$. Then the ultraweak closure of $Q_{0}$ is $Q$.

Density in the other topologies follows once one is reminded that the ultrastrong and ultraweak closures of convex sets of operators are the same.

For the study of commutative subspace lattices, there is available a very useful tool: Arveson's spectral representation theorem. Let $X$ be a compact metric space, let $\leqslant$ be a reflexive and transitive relation on $X$ whose graph $G$ is a closed subset of $X \times X$, and let $m$ be a finite Borel measure on $X$. A Borel subset $S \subseteq X$ is said to be increasing if $x \in S$ and $x \leqslant y$ imply $y \in S$. For each Borel subset $S$ of $X$ let $P_{s}$ denote the corresponding orthogonal projection acting on the Hilbert space $\mathcal{H}=$ $L^{2}(X, m)$, i.e., $P_{s} f(x)=\chi_{s}(x) f(x)$ for $f \in L^{2}(X, m)$. Let $\mathcal{L}(X, \leqslant, m)=\left\{P_{s} \mid S\right.$ is an increasing Borel subset of $X$ \}. Arveson's theorem [1, Theorem 1.3] asserts that every commutative subspace lattice acting on a separable Hilbert space is unitarily equivalent to some $\mathcal{L}(X, \leqslant, m)$. For $\mathcal{L}=\mathcal{L}(X, \leqslant, m)$, there is a (measure theoretic) characterization of complete distributivity involving $G$, the graph of $\leqslant[5$, Theorem 7]:

A commutative subspace lattice $\mathcal{L}=\mathcal{L}(X, \leqslant, m)$ is completely distributive if and only if, for a Borel set $A$ with $m(A)>0$, we have $m \times m(A \times A \cap G)>0$.

By an integral operator on $L^{2}(X, m)$ we will mean a bounded operator $T$ for which there exists a Borel measurable function $k$ on $X \times X$ such that for any $f, g \in L^{2}(X, m)$ we have

$$
(T f, g)=\int k(x, y) f(y) \overline{g(x)} d m \times m(x, y) .
$$


THEOREM 4. Let $\mathcal{L}=\mathfrak{L}(X, \leqslant, m)$ be a commutative subspace lattice. Suppose there exists a subset $\mathbb{Q}_{0}$ of integral operators in $\mathrm{Alg} \varrho$ which is dense in $\mathrm{Alg} \mathcal{E}$ in any of the strong, weak, ultrastrong or ultraweak topologies. Then $\mathcal{L}$ is completely distributive and the rank-one subalgebra of $\mathrm{Alg} \mathcal{L}$ is dense in $\mathrm{Alg} \mathcal{E}$ in any of these topologies.

Proof. The last clause of the conclusion follows from Theorem 3 once we show that $\mathcal{L}$ is completely distributive. Assume we have a subset $\mathbb{Q}_{0}$ of $\operatorname{Alg} E$ consisting of integral operators which is dense in $\mathrm{Alg} \mathcal{E}$ in any of the mentioned topologies. This implies that, for any nonzero projection $P$ on $\mathcal{H}$ there exists $T$ in $Q_{0}$ such that $P T P \neq 0$. (This follows since the density of $\Theta_{0}$ in Alg $£$ implies that there exists a net $\left\{T_{\nu}\right\}$ in $\mathbb{Q}_{0}$ such that $T_{\nu} \rightarrow I$ and hence $P T_{\nu} P \rightarrow P \neq 0$.) For any $T \in \mathcal{Q}_{0}$ there is an associated kernel function $k$. Using the facts that $T \in A \operatorname{Alg} \&$ and that an integral operator on an $L^{2}$ space is zero iff the kernel is zero a.e. [3, Theorem 8.1], we can conclude that $k$ lives on $G$ a.e. following the proof of [1, Proposition 1.6.0].

Let $A$ be a Borel set such that $m(A)>0$ and let $P$ be the projection corresponding to multiplication by $\chi_{A}$. By the previous paragraph there exists a $T \in \mathbb{Q}_{0}$ such that $P T P \neq 0$. Let $k$ be the kernel function for $T$. There exist $f, g \in L^{2}(X, m)$ which live on $A$ such that

$$
0 \neq(T f, g)=\int k(x, y) f(y) \overline{g(x)} d m \times m(x, y)
$$

But this implies that $m \times m(A \times A \cap G)>0$ since $k(x, y)$ lives on $G$ and $f(y) \overline{g(x)}$ lives on $A \times A$. This in turn implies complete distributivity by [5, Theorem 7].

REMARKS. (1) Even though the subalgebra of finite rank operators in Alg $£$ may be bigger than the rank-one subalgebra (see [4]), density of the finite ranks implies density of the rank-one subalgebra.

(2) Theorem 4 can be thought of as giving a family of characterizations of complete distributivity for complete subspace lattices.

(3) Theorem 4 does not hold for noncommutative subspace lattices. Lambrou [7] gives an example of a lattice in which the subalgebra of the finite rank operators is strongly dense but the lattice is not completely distributive.

Problem 1. It is still undecided whether complete distributivity of a noncommutative subspace lattice $\mathcal{L}$ implies the density of the rank-one subalgebra in $\operatorname{Alg} \mathcal{E}$.

Problem 2. Let $C_{p}$ denote the Schatten $p$-class of compact operators. Theorem 4

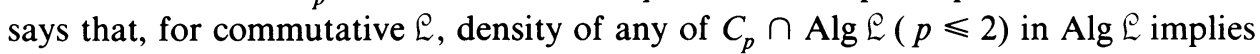
density of the rank-one subalgebra of $\operatorname{Alg} \mathcal{L}$. Does this hold for $C_{p} \cap \operatorname{Alg} \mathcal{L}, p>2$, or (of ultimate interest) does it hold for the subalgebra of compact operators in Alg $\mathcal{L}$ ?

\section{REFERENCES}

1. W. Arveson, Operator algebras and invariant subspaces, Ann. of Math. (2) 100 (1974), 433-532.

2. J. Erdos, Operators of finite rank in nest algebras, J. London Math. Soc. 43 (1968), 391-397.

3. P. R. Halmos and V. S. Sunder, Bounded integral operators on $L^{2}$ spaces, Springer-Verlag, Berlin and New York, 1978. 
4. A. Hopenwasser and R. Moore, Finite rank operators in reflexive operator algebras, J. London Math. Soc. (to appear).

5. A. Hopenwasser, C. Laurie and R. Moore, Reflexive algebras with completely distributive subspace lattices, J. Operator Theory (to appear).

6. M. S. Lambrou, Completely distributive lattices, Fund. Math. (to appear).

7. Approximants, commutants, and double commutants in normed algbebras, J. London Math. Soc. (2) 25 (1982), 499-512.

8. C. Laurie, On density of compact operators in reflexive algeghras, Indiana Univ. Math. J. 30 (1981), $1-16$.

9. W.E. Longstaff, Strongly reflexive lattices, J. London Math. Soc. (2) 11 (1975), 491-498.

10. __ Operators of rank one in reflexive algebras, Canad. J. Math. 28 (1976), 19-23.

Department of Mathematics, University of Alabama, University, Alabama 35486

Department of Mathematics, University of Western Australia, Nedlands, Western Australia 6009, Australia 